\title{
命HAD
}

DOI: http://doi.org/10.22585/hospdomic.v6i1.152

\section{Estudio epidemiológico de la prevalencia del síndrome de intestino corto en España}

\section{Epidemiological study of the prevalence of short bowel syndrome in Spain}

\author{
Carmina Wanden-Berghe' (1) 0000-0002-6871-5737 \\ Vanessa E. Oller-Arlandis² (1) 0000-0003-4944-3796 \\ Andrea Domingo-Pueyo² (1) 0000-0001-5978-0679 \\ 1. Hospital General Universitario de Alicante. ISABIAL-FISABIO, Alicante, España. \\ 2. Centro Internacional Virtual de Investigación en Nutrición (CIVIN), Alicante, España.
}

Correspondencia/Correspondence

Carmina Wanden-Berghe

carminaw@telefonica.net

Recibido/Received

24.12.2021

Aceptado/Accepted

10.01 .2022
Conflicto de Intereses/Competing interest

Las autoras declaran la inexistencia de conflicto de interés.

Financiación/Funding

No se han recibido fuentes de financiación.

Contribuciones de autoría/Author contributions

Las autoras declaran la inexistencia de conflicto de interés. 


\section{RESUMEN}

Introducción: El síndrome de intestino corto (SIC) es una alteración de la absorción de los nutrientes causado, frecuentemente, por la resección quirúrgica del intestino delgado. El espectro de las manifestaciones clínicas varía ampliamente, según la longitud remanente del intestino delgado.

Objetivo: Conocer la prevalencia de los casos de SIC en España en el año 2018.

Método: Estudio observacional transversal multicéntrico, de base poblacional, mediante cuestionario telemático en el que se recogen los datos de los pacientes diagnosticados de SIC en 2018.

Resultados: De un total de 541 centros sanitarios seleccionados, del Catálogo Nacional de Hospitales del Ministerio de Sanidad, participaron 101 hospitales. Con los datos obtenidos, la prevalencia de SIC en España se estimaría en 4,18 pacientes por millón de habitantes durante ese año.

Conclusiones: La falta de participación dificulta obtener resultados concluyentes, lo que resulta mucho más importante cuando se trata de problemas de salud poco prevalentes.

Palabras clave: Síndrome del Intestino Corto; Nutrición Parenteral en el Domicilio; Servicios de Atención de Salud a Domicilio; Epidemiología; Prevalencia.

\section{ABSTRACT}

Introduction: Short bowel syndrome (SBS) is a disturbance in nutrient absorption often caused by surgical resection of the small intestine. The spectrum of clinical manifestations varies widely, depending on the remaining length of the small intestine.

Objective: To estimate the prevalence of cases of SBS cases in Spain in 2018.

Method: A population-based, multicenter crosssectional study, using an online survey, that collects data from patients diagnosed with SBS in 2018.

Results: From a total of 541 health centers selected, of the National Catalog of Hospitals of the Ministry of Health, 101 hospitals took part in. With the data obtained, the prevalence of SIC in Spain would be estimated at 4.18 patients per million inhabitants during that year.

Conclusions: Lack of participation makes it difficult to obtain conclusive results, which is much more important when it comes to low prevalence health topics.

Keywords: Short Bowel Syndrome; Parenteral Nutrition, Home; Home Care Services; Epidemiology; Prevalence. 


\section{INTRODUCCIÓN}

El síndrome del intestino corto (SIC) se define como la pérdida o ausencia de enterocitos o de masa intestinal causada por resección quirúrgica, defectos congénitos o cualquier enfermedad que pueda causar un defecto de la función intestinal ${ }^{(1)}$. La longitud de intestino funcional necesaria para definirlo no es exacta, pero parece haber consenso en menos de $200 \mathrm{~cm}$. De estos pacientes, un subgrupo presentará insuficiencia intestinal, con incapacidad para mantener un equilibrio hídrico y nutricional con la alimentación entérica, siendo necesaria la fluidoterapia y/o la nutrición parenteral (NP). Esto ocurre cuando el yeyuno es inferior a $35 \mathrm{~cm}$ con anastomosis yeyuno-ileal, a $60 \mathrm{~cm}$ con anastomosis yeyuno-cólica y a $150 \mathrm{~cm}$ con yeyunostomías terminales ${ }^{(2)}$. Mientras que los pacientes con y sin colon tienen problemas con la absorción de nutrientes, aquellos con yeyunostomía también tienen problemas de pérdidas de agua, sodio y magnesio. Las pérdidas por el estoma pueden exceder la ingesta oral y todos estos pacientes necesitan reposición hidroelectrolítica parenterales. Las pérdidas de líquidos y sodio pueden reducirse con octreótido, omeprazol o bloqueadores H2, pero no lo suficiente como para evitar la necesidad de reposición intravenosa ${ }^{(3)}$.

El concepto de la atención domiciliaria del paciente con NP ha evolucionado en las últimas décadas, gran parte de la atención que precisan estos pacientes se ofrece principalmente en el hogar, es importante que contemplemos este servicio como crucial en su evolución ${ }^{(4-6)}$. Otro factor que afecta, o puede afectar, en la evolución de los pacientes con SIC es el tratamiento farmacológico que recientemente ha comenzado a tener un impacto significativo en el cuidado y el manejo nutricional de estos pacientes, de forma que surge la posibilidad de reducir las necesidades de NP en pacientes que antes dependían de ella.

La prevalencia e incidencia de este síndrome es difícil de conocer, en parte por la falta de acuerdo sobre su definición y en parte, por las diferentes especialidades y modalidades de seguimiento que presentan. Se suele estimar indirectamente a través del número de pacientes que reciben nutrición parenteral domiciliaria (NPD), reflejados anualmente en las publicaciones del Grupo de trabajo NADYA (Nutrición Artificial Domiciliaria y Ambulatoria) de la sociedad española de nutrición clínica y metabolismo. Las estimaciones publicadas sobre la prevalencia indican que el SIC afecta a tres personas de cada millón en EE. UU. y de forma similar en Europa y en España ${ }^{(7-9)}$. Aunque de estos cálculos se escapan los pacientes que no requieren NP, pero necesitan líquidos y electrólitos y, también, los que se mantienen con estrategias de tratamiento enteral.

En este trabajo se ha planteado una encuesta dirigida a los diferentes servicios hospitalarios directamente relacionados con la atención del SIC, con el propósito de llegar a aproximarnos al conocimiento de la prevalencia durante el año estudiado.

El objetivo principal fue evaluar la prevalencia deI SIC en la población y secundariamente:

- Conocer la distribución geográfica de la incidencia del SIC en España.

- Comparar la prevalencia de SIC entre las distintas regiones del territorio español.

- Describir las características de los pacientes con SIC.

\section{MÉTODOS}

\section{Diseño}

Estudio observacional de corte transversal multicéntrico, de base poblacional. 


\section{Método}

Mediante encuesta desarrollada ad hoc. Se entrevistó a los diferentes servicios implicados en la atención sanitaria de los pacientes con SIC de los hospitales españoles registrados en el catálogo nacional de hospitales (CHN) del Ministerio de Sanidad, actualizado a $2018^{(10)}$. Se registraron los casos diagnosticados de SIC a lo largo del año 2018 en España.

Criterios de inclusión y exclusión: se excluyeron los hospitales que no se adecuaron a los objetivos del proyecto principalmente porque su cartera de servicios no posibilitaba que se atendiera pacientes con SIC. Se eliminaron los que cumplieron los siguientes criterios de finalidad asistencial: hospitales geriátricos y/o de larga estancia, penitenciarios, psiquiátricos y monográficos (cirugía estética, osteoarticular).

Se contactó telefónicamente con los hospitales seleccionados, durante el periodo comprendido desde el 1 de abril de 2019 hasta el 31 de agosto de 2020, para solicitar su colaboración en el estudio mediante un primer contacto con los profesionales sanitarios responsables de los distintos servicios, tanto de farmacia hospitalaria como de gastro-entereología o endocrinología y nutrición, como aquellos que pudieran estar implicados en el tratamiento y/o seguimiento del SIC. Posteriormente, se remitió, vía correo electrónico, el enlace a la encuesta electrónica para su cumplimentación online, y se reenviaron correos semanales, a forma de recordatorio, durante 4 semanas consecutivas.

\section{Análisis estadístico}

La población de referencia del estudio estuvo constituida por la población de las áreas de los centros participantes en el estudio. Se calculó la tasa de prevalencia (número de casos por millón de habitantes durante el año 2018) y las tasas de prevalencia estandarizada por comunidad autónoma. Para el cálculo de las prevalencias se tomó como denominador el dato de población proporcionado por el Instituto Nacional de Estadística a 1 de enero de 2018 (46.658.447 habitantes) y los correspondientes por comunidad autónoma ${ }^{(11)}$.

En las variables cualitativas se calcularon los porcentajes (con sus intervalos de confianza del $95 \%$ ) y en las variables cuantitativas la media aritmética y la desviación estándar.

El almacenamiento y análisis estadístico se realizó con el programa Statistical Package for the Social Sciences (IBM-SPSS), versión 25 para Windows®.

\section{Aspectos éticos}

El estudio se llevó a cabo siguiendo las recomendaciones éticas internacionales para la investigación clínica en humanos recogidas en la Declaración de Helsinki de 1964 y sus sucesivas actualizaciones, y siguiendo las recomendaciones del Ministerio de Sanidad. Fue aprobado por el Comité de Ética e Investigación Clínica (CEIC) antes de su inicio.

\section{RESULTADOS}

Un total de 541 hospitales del CNH del Ministerio de Sanidad -año 2018- cumplían con los criterios para ser incluidos en el presente trabajo. De ellos, 77 (14,23\%) hospitales contactados manifestaron, en un primer contacto telefónico, no querer participar en el estudio por los siguientes motivos: $11(14,29 \%)$ son únicamente depósito/botiquín de farmacia; otros 18 (23,38\%) hospitales no tienen posibilidad de preparar o dispensar bolsas de NP; otros $8(10,39 \%)$ indicaron que, por 
su finalidad asistencial, no tienen cirugías digestivas o servicio de endocrinología y nutrición (o análogos) y, finalmente, 37 (48,05\%) profesionales sanitarios manifestaron, abiertamente, no querer participar en ningún proyecto o encuesta. Destacar, además, que en $3(3,90 \%)$ centros, el personal con el que se estableció contacto, manifestaron que debido a la situación de pandemia Covid-19 el hospital/clínica estaba cerrado (cese temporal de actividad) y toda la atención sanitaria había sido centralizada en el correspondiente hospital de referencia de la provincia.

Por último, señalar que hubo 15 (2,77\%) hospitales cuyo contacto telefónico fue infructuoso, tras numerosos intentos en diferentes horarios y días. Por tanto, de los $449(82,99 \%)$ centros sanitarios que aceptaron participar, se han obtenido 101 (18,67\%; IC95\% 15,39 - 21,95) cuestionarios cumplimentados. En la figura 1 se muestra el porcentaje de participación de los centros según el número de camas.

\section{Figura 1.- Porcentaje de participacion de los centros por número de camas}

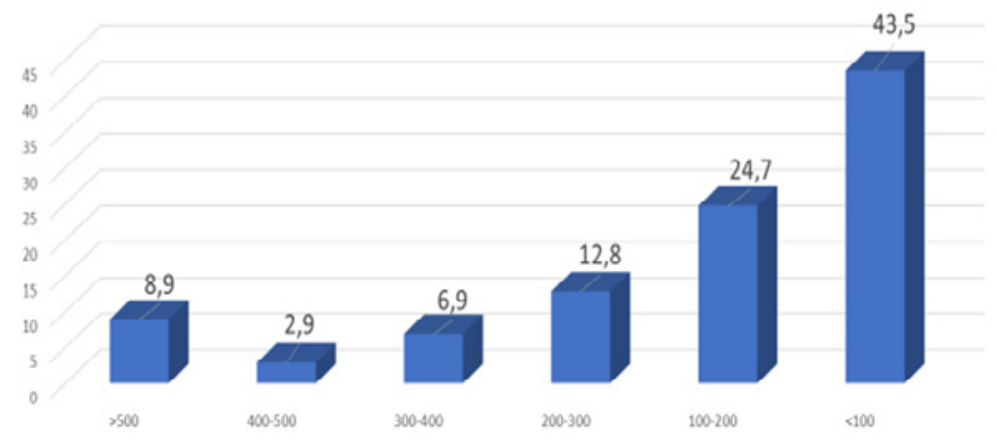

De los 101 hospitales que formaron parte de este estudio, la mayoría de los cuestionarios, 95, fueron cumplimentados por profesionales que pertenecían al servicio de farmacia (94,06\%; IC95\% 89,45 - 98,67) (tabla 1). Los centros más participativos fueron los de la provincia de Barcelona con 12 centros hospitalarios (11,88\%; IC 95\% 5,57 - 18,19), ver figura 2 .

Tabla 1. Especialidades que han colaborado en el estudio

\begin{tabular}{|l|l|l|}
\hline & Hospitales $\sin$ NP (\%) & Hospitales con NP (\%) \\
\hline Farmacia hospitalaria & $13(86,66 \%)$ & $82(95,35 \%)$ \\
\hline Endocrinología y Nutrición & - & $3(3,49 \%)$ \\
\hline
\end{tabular}




\begin{tabular}{|l|l|l|}
\hline $\begin{array}{l}\text { Unidad Hospitalización a } \\
\text { Domicilio }\end{array}$ & - & $1(1,16 \%)$ \\
\hline Enfermería & $1(6,66 \%)$ & - \\
\hline Medicina general & $1(6,66 \%)$ & - \\
\hline Total & $\mathbf{1 5 ( 1 4 , 8 5 \% )}$ & $\mathbf{8 6}(\mathbf{8 5 , 1 5 \% )}$ \\
\hline
\end{tabular}

Figura 2.- Distribución geográfica del Sindrome de Intestino Corto

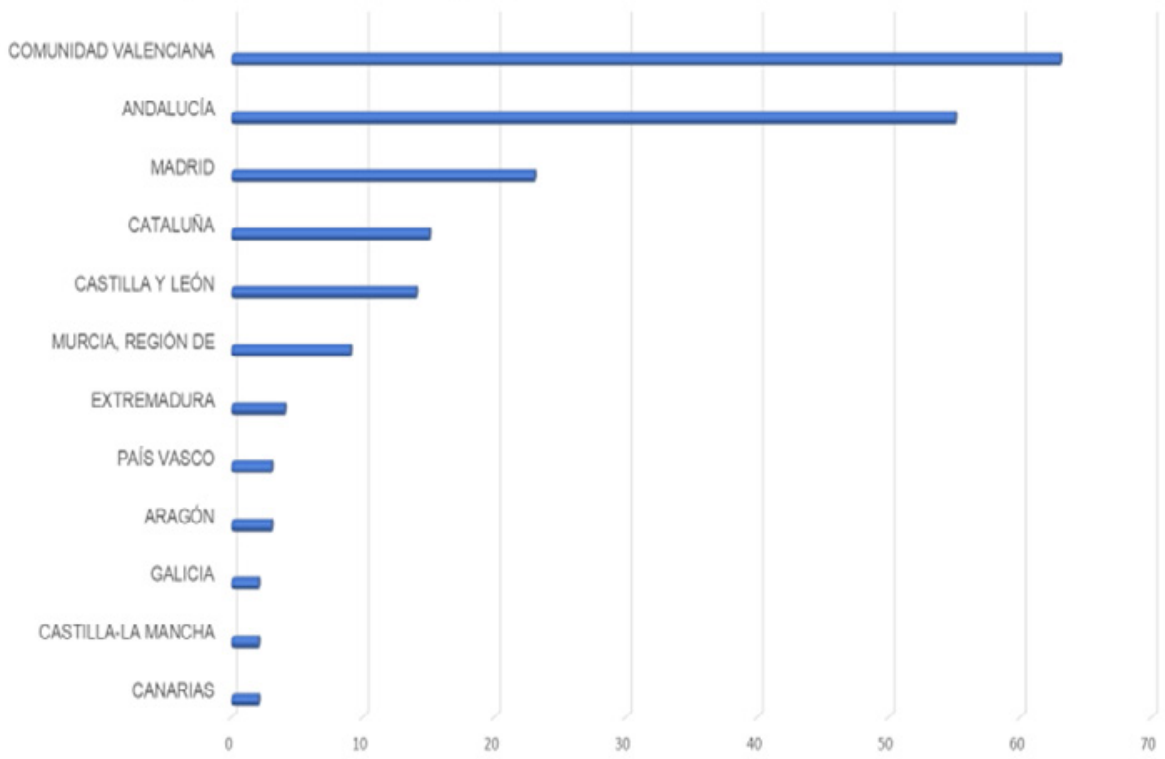

Con respecto a las variables demográficas de los pacientes objeto de estudio, la edad se obtuvo en 31 de ellos, siendo 27 (87,1\%; IC 95\% 75,30 - 98,90) adultos con 60,07 \pm 13 , 49 años y 4 (12,9\%; IC 95\% 1,10 - 24,70) niños de $9 \pm 2,16$ años de edad media. Se obtuvo información sobre el género de 20 individuos, siendo en su mayoría mujeres 14 vs 6 (70,00\%; IC 95\% 49,92 - 90,08).

De los 101 hospitales que cumplimentaron la encuesta, 86 (85,15\%; IC 95\% 78,21 - 92,08) declararon administrar nutriciones parenterales en sus hospitales de forma habitual, si bien, 16 (18,60\%; IC 95\% 10,38 - 26,83) centros declararon no haber administrado ninguna durante ese año.

Conforme a las encuestas cumplimentadas, en el año 2018, recibieron NP 9840 pacientes, una media de 126, $15 \pm 206,33$ por centro (mín. 1 - máx. 1095). Los pacientes nuevos registrados fueron 9109, con una media de 116,77 $\pm 182,65$, la mayor incidencia por hospital fue 875 pacientes.

El motivo de la indicación de la NP fue en 196 pacientes (1,99\%; IC95\% 1,72 - 2,27) por presentar SIC, de los que 85 (43,37\%; IC95\% 36,43 - 50,31) fueron funcionales y 105 (53,57\%; IC95\% 46,59 - 60,55) quirúrgicos, y en 6 de los casos no se pudo obtener información. De los pacientes con resección intestinal, 94 (89,52\%; IC95\% 83,67 - 95,38) conservaban el colon o parte de él, 84 
(80,00\%; IC95\% 72,35 - 87,65) mantenían válvula ileocecal y 54 (51,43\%; IC95\% 41,87\% - 60,99) eran portadores de ostomía. Los pacientes tratados con análogos del GLP-2 fueron 15 (14,29\%; IC95\% 7,59 - 20,98).

En el momento de realizar el estudio 162 pacientes (82,65\%; IC95\% 77,35 - 87,95) se encontraban dados de alta, de los que 32 (19,75\%; IC95\% 13,62 - 25,88) necesitaron NPD y 65 (40,12\%; IC95\% 32,58 - 47,67) reposición hidroelectrolítica en su domicilio.

Con los datos obtenidos, la prevalencia de SIC en España se estimaría en 4,18 pacientes por millón de habitantes durante el año 2018. Las estimaciones de las prevalencias por comunidades autónomas se muestran en la tabla 2.

Tabla 2. Estimación de la prevalencia del SIC por comunidad autónoma

\begin{tabular}{|l|l|}
\hline & $\begin{array}{l}\text { Prevalencia } \\
(1.000 .000 \text { hab./año })\end{array}$ \\
\hline Andalucía & 6,54 \\
\hline Aragón & 2.28 \\
\hline Canarias & 0,92 \\
\hline Castilla y León & 5,79 \\
\hline Castilla-La Mancha & 0,98 \\
\hline Cataluña & 2,00 \\
\hline Comunidad Valenciana & 12,73 \\
\hline Extremadura & 3,74 \\
\hline Galicia & 0,74 \\
\hline Madrid & 3,51 \\
\hline Comunidad de Murcia & 6,10 \\
\hline Región de País Vasco & 1,38 \\
\hline España & 4,18 \\
\hline
\end{tabular}




\section{DISCUSIÓN}

Actualmente, la prevalencia real de SIC en todo el mundo no se conoce completamente debido, principalmente, a la ausencia de bases de datos fiables ${ }^{(1,12)}$. Las mejores estimaciones se basan en el número de pacientes que reciben nutrición parenteral a largo plazo, recogidos en los informes de NPD y/o soporte de líquidos por vía intravenosa. Estos registros estiman que el 37,3\% de las NPD están motivadas por SIC ${ }^{(13)}$. También, se ha informado que la prevalencia de SIC es de 1,4 casos por millón de habitantes en Europa (que van desde 0,4 en Polonia a 40 en Dinamarca) y 30 casos por millón en los EE. UU.(12,14,15). En el presente estudio, la prevalencia estimada se situaría en el rango inferior de las informadas en Europa. Este hecho coincide con las prevalencias de NPD que periódicamente informa el grupo NADYA que, también, se mueven en rangos bajos respecto al resto de países europeos ${ }^{(13)}$.

EI SIC con fallo intestinal, por su baja prevalencia, se considera una enfermedad rara. En España, los primeros casos publicados en pacientes adultos datan de finales de los años ochenta y en niños de mediados de los noventa ${ }^{(16)}$. Pero no todos los pacientes con SIC están destinados a una insuficiencia intestinal ya que, con los tratamientos actuales, esta condición puede ser reversible. Entonces, ¿puede ser considerado realmente el SIC como una enfermedad rara en España? Desafortunadamente, debido a la escasa tasa de respuesta obtenida en este proyecto, por parte de los centros sanitarios seleccionados del $\mathrm{CHN}$, no se puede presuponer de antemano este concepto ya que, como concluyó un estudio alemán, con una tasa de respuesta global a la encuesta del $85 \%$, los datos de prevalencia obtenidos (34 pacientes por millón de habitantes) indicaban una aparición más frecuente del cuadro clínico de SIC de lo que se presuponía basándose en datos de NPD y, por ende, no se podía considerar el SIC como una enfermedad rara ${ }^{(17)}$. En este sentido, no se puede descartar que el número real de pacientes en España esté infraestimado debido a la escasa cooperación de los centros seleccionados. A pesar de ello, cabe destacar que, en España, el síndrome de intestino corto congénito consta como una enfermedad catalogada en el «Registro de Pacientes de Enfermedades Raras del Instituto de Salud Carlos III»(18).

En cuanto a las características demográficas de los pacientes SIC, señalar que, respecto a la distribución por género, algunos autores han encontrado una prevalencia más elevada en mujeres que en varones, posiblemente debido a la longitud más corta del intestino delgado en mujeres ${ }^{(19)}$. De hecho, Underhill (1955) informó sobre la longitud del intestino delgado en varones en un rango entre 488 - 785 (media 637) cm y en mujeres de $335-716$ (media de 592) $\mathrm{cm}^{(20)}$. En este sentido, un reciente estudio internacional multicéntrico, en el que también había reclutados 21 pacientes españoles señaló, en adultos, una ratio de $2: 1^{(21)}$. Lamentablemente en este trabajo, los datos respecto al sexo solo se han informado en un número muy escaso de pacientes sin que haya permitido sacar conclusiones al respecto.

Otro aspecto epidemiológico relevante es la edad de los pacientes. En referencia a este dato, este estudio no individualiza a los pacientes, sino que pretende aportar datos de prevalencia conjunta. Pero, dado que en los cuestionarios respondidos este parámetro se ha cumplimentado en muy pocas ocasiones, la escasez de la muestra, sobre todo pediátrica, tampoco permitiría extraer conclusiones válidas.

Finalmente, como alternativa a los periodos de larga hospitalización, las nuevas estrategias de tratamiento en NPD representan un método terapéutico seguro y eficaz para obtener un adecuado estado nutricional, evitar complicaciones y mejorar la calidad de vida de los pacientes y, también, de sus familias y cuidadores ${ }^{(16,22)}$. Así, algunos estudios llevados a cabo en diferentes países europeos, sobre la prevalencia de la NPD en niños con insuficiencia intestinal crónica, han informado cifras en los < 18 años de 9,56 casos por millón de habitantes, como es el caso de los Países Bajos en 2013 o de Italia con una prevalencia en los $\leq 19$ años de 14,12 pacientes por millón de habitantes, 
siendo en su mayoría (58\%) causados por SIC ${ }^{(22,23)}$. En 2019, un estudio en Reino Unido publicó una prevalencia de NPD de 30 niños por millón, el 48\% de ellos estaban diagnosticados de SIC(24). En España, el último informe publicado del grupo NADYA, en 2019, presentó datos de NPD siendo el SIC el primer motivo de indicación terapéutica tanto en niños $(51,6 \%)$ como en adultos $(37,3 \%)^{(13)}$.

Limitaciones: la principal limitación del estudio ha residido en la baja colaboración/respuesta de los centros contactados, lo que no ha permitido disponer de un número amplio de datos que posibiliten extraer conclusiones. Este problema junto a la pandemia de la Covid-19 han sido las mayores dificultades encontradas.

Se puede concluir que: la falta de participación dificulta obtener resultados concluyentes, lo que resulta mucho más importante cuando se trata de problemas de salud poco prevalentes. Con este trabajo se pretendió, por primera vez en España, obtener datos empíricos sobre la prevalencia del SIC en la población general, ya que, a día de hoy, no hay datos epidemiológicos validados que permitan conocer la situación real de esta enfermedad.

Sería interesante que con objeto de poder determinar la prevalencia y la incidencia del SIC, se estableciera un registro estatal obligatorio de pacientes con SIC para una comunicación precisa de los casos que sirva de herramienta en la toma de decisiones de futuras estrategias de salud.

\section{BIBLIOGRAFÍA}

1. O'Keefe SJ, Buchman AL, Fishbein TM, Jeejeebhoy KN, Jeppesen PB, Shaffer J. Short bowel syndrome and intestinal failure: consensus definitions and overview. Clin Gastroenterol Hepatol. 2006;4(1):6-10. DOI: 10.1016/j.cgh.2005.10.002

2. Carbonnel F, Cosnes J, Chevret S, Beaugerie L, Ngô Y, Malafosse M, et al. The role of anatomic factors in nutritional autonomy after extensive small bowel resection. JPEN J Parenter Enteral Nutr. 1996;20(4):275-80. DOI: 10.1177/0148607196020004275

3. Nightingale JM, Lennard-Jones JE. The short bowel syndrome: what's new and old? Dig Dis. 1993;11(1):12-31. DOI: 10.1159/000171397

4. Mullady DK, O'Keefe SJ. Treatment of intestinal failure: home parenteral nutrition. Nat Clin Pract Gastroenterol Hepatol. 2006;3(9):492-504. DOI: 10.1038/ncpgasthep0580

5. Nightingale J, Woodward JM, Gastroenterology SBaNCotBSo. Guidelines for management of patients with a short bowel. Gut. 2006;55(Suppl 4):1-12. DOI: 10.1136/gut.2006.091108

6. Carroll RE, Benedetti E, Schowalter JP, Buchman AL. Management and Complications of Short Bowel Syndrome: An Updated Review. Curr Gastroenterol Rep. 2016;18(7):e40. DOI: 10.1007/ s11894-016-0511-3

7. Pironi L, Goulet O, Buchman A, Messing B, Gabe S, Candusso M et al. Outcome on home parenteral nutrition for benign intestinal failure: a review of the literature and benchmarking with the European prospective survey of ESPEN. Clin Nutr. 2012;31(6):831-45. DOI: 10.1016/j. clnu.2012.05.004

8. Higuera I, García-Peris P, Camblor M, Bretón I, Velasco C, Romero R, et al. Outcomes of a general hospital-based home parenteral nutrition (HPN) program; report of our experience from a 26-year period. Nutr Hosp. 2014;30(2):359-65. DOI: 10.3305/nh.2014.30.2.7592

9. Howard L. Home parenteral nutrition: survival, cost, and quality of life. Gastroenterology. 2006;130(Suppl 2):S52-9. DOI: 10.1053/j.gastro.2005.09.065 
10. Fundación Instituto para el Desarrollo e Integración de la Sanidad (IDIS). Informe Sanidad Privada: Aportando Valor. Análisis de situación 2014 [Internet]. Madrid, España: IDIS; 2014 [consultado 5 noviembre de 2021]. Disponible en: https://bit.ly/3pyEgyt

11. Instituto Nacional de Estadística (INE). INEBASE (sede Web). Madrid, España: INE; 2016 [consultada 5 noviembre de 2021]. Disponible en: https://bit.ly/3Es1pGX

12. Buchman AL, Scolapio J, Fryer J. AGA technical review on short bowel syndrome and intestinal transplantation. Gastroenterology. 2003;124(4):1111-34. DOI: 10.1016/s0016-5085(03)70064-x

13. Wanden-Berghe C, Virgili Casas N, Cuerda Compes C, Ramos Boluda E, Pereira Cunill JL, Maiz Jiménez MI, et al.; Grupo NADYA-SENPE. Nutrición parenteral domiciliaria en España, 2019: informe del Grupo de Nutrición Artificial Domiciliaria y Ambulatoria NADYA. Nutr Hosp. 2021;38(6):1304-9. DOI: 10.20960/nh.03720

14. DiBaise JK, Young RJ, Vanderhoof JA. Intestinal rehabilitation and the short bowel syndrome: part 1. Am J Gastroenterol. 2004;99(7):1386-95. DOI: 10.1111/j.1572-0241.2004.30345.x

15. Jeppesen PB. Teduglutide, a novel glucagon-like peptide 2 , in the treatment of patients with short bowel syndrome. Therap Adv Gatroenterol. 2012;5(3):159-71. DOI: 10.1177/1756283X11436318

16. González Aguilera B, Olveira G, García-Luna PP, Pereira Cunill JL, Luengo LM, Pérez de la Cruz A, Irles Rocamora JA. Documento de Consenso de expertos de Andalucía y Extremadura sobre la nutrición parenteral domiciliaria. Nutr Hosp. 2017;34(4):784-91. DOI: 10.20960/nh.881

17. von Websky MW, Liermann U, Buchholz BM, Kitamura K, Pascher A, Lamprecht G, et al. Short bowel Syndrome in Germany: Estimated prevalence and standard of care. Chirurg. 2014;85(5):433-9. DOI: 10.1007/s00104-013-2605-x

18. Instituto de Salud Carlos III (ISCIII). Portal de registro de enfermedades raras [Internet]. Madrid, España: ISCIII; [consultado 12 diciembre 2021]. Disponible en: https://bit.ly/3qqxKZt

19. Nightingale JM, Lennard-Jones JE, Gertner DJ, Wood SR, Bartram Cl. Colonic preservation reduces need for parenteral therapy, increases incidence of renal stones, but does not change high prevalence of gall stones in patients with a short bowel. Gut. 1992;33(11):1493-7. DOI: 10.1136/gut.33.11.1493

20. Underhill BM. Intestinal length in man. Br Med J. 1955;19:2(4950):1243-6. DOI: 10.1136/ bmj.2.4950.1243

21. Pironi L, Steiger E, Joly F, Jeppesen PB, Wanten G, Sasdelli AS, et al. Characteristics of adult patients wirh chronic intestinal failure due to short bowel syndrome: An international multicenter survey. Clin Nutr ESPEN. 2021;45:433-44. DOI: 10.1016/clnesp.2021.07.004

22. Neelis EG, Roskott AM, Dijkstra G, Wanten GJ, Serlie MJ, Tabbers MM, et al. Presentation of a nationwide multicenter registry of intestinal failure and intestinal transplantion. Clin Nutr. 2016;35(1):225-9. DOI: 10.1016/clnu.2015.01.010

23. Diamanti A, Capriati T, Gandullia P, Di Leo G, Lezo A, Lacitignola L, et al. Pediatric Chronic Intestinal Failure in Italy: Report from the 2016 Survey on Behalf of Italian Society for Gastroenterology, Hepatology and Nutrition (SIGENP). Nutrients. 2017;9(11):1217. DOI: 10.3390/nu9111217

24. Wiskin AE, Russell R, Barclay AR, Thomas J, Batra A; BANS committee of BAPEN. Prevalence of home parenteral nutrition in children. Clin Nutr ESPEN. 2021;42:138-41. DOI: 10.1016/J.CLNESP.2020.12.029 